\title{
Telemedizin: „TEMPiS“-Netzwerk für Schlaganfallbehandlung in der Regelversorgung
}

Nicolas Völkel, Gordian J. Hubert, Roman L. Haberl

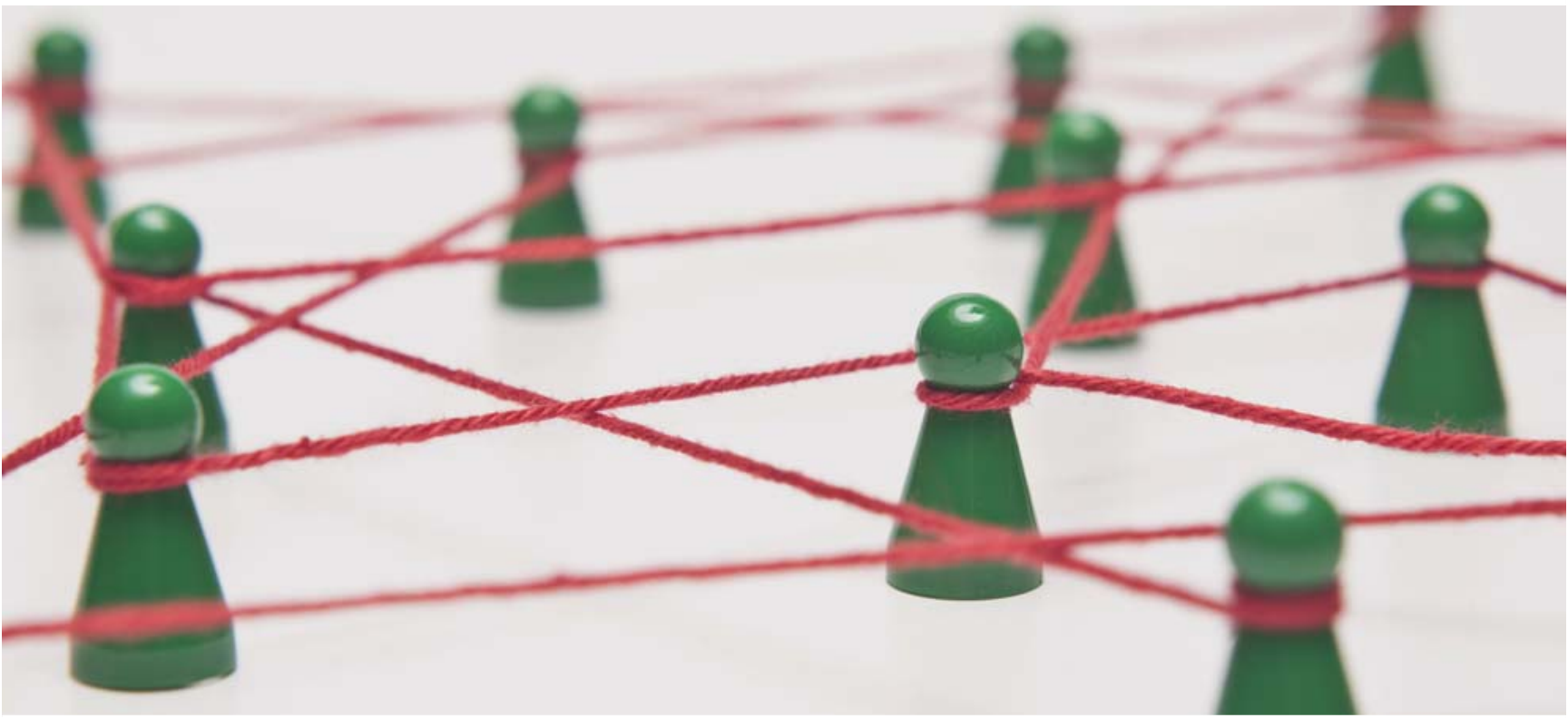

- Abb. 1 Quelle: rockstar images/Fotolia.

Bei einem Schlaganfall muss es schnell gehen: Thrombolyse und Thrombektomie sind hochgradig zeitkritisch - in jeder Minute ohne Behandlung sterben im Durchschnitt fast 2 Mio. Nervenzellen ab. Dieser Beitrag schildert, wie telemedizinische Netzwerke - insbesondere in ländlichen Regionen - die Versorgungsstrukturen für Schlaganfallpatienten signifikant verbessern und das Risiko einer bleibenden Behinderung senken.

\section{Hintergrund}

Trotz aller Anstrengungen und Erfolge der letzten Jahrzehnte ist der Schlaganfall immer noch eine Erkrankung, die eine große Herausforderung darstellt. Ungefähr 260000 Schlaganfälle ereignen sich in Deutschland pro Jahr. Mit jährlich etwa 63000 Todesfällen steht der Schlaganfall an 3. Stelle der Todesursachen. Hinsichtlich der Anzahl von Patienten mit bleibenden Behinderungen steht er sogar an 1. Stelle: Ungefähr eine Million Menschen leben in Deutschland mit den Folgen eines Schlaganfalls [1].

Aufgrund des demografischen Wandels ist anzunehmen, dass diese Zahlen zukünftig noch weiter steigen. Daher wird es eine dringliche Aufgabe, eine angemessene Ver- sorgungsqualität für Schlaganfallpatienten zu gewährleisten. Besonders in ländlichen Regionen ist spezialisiertes Personal - insbesondere die vaskulären Neurologen nicht ausreichend vorhanden. Es gilt also ein Konzept zu entwickeln, das Versorgungsstrukturen derart aufbaut, dass alle Patienten mit einem Schlaganfall in Deutschland eine optimale Therapie erhalten können.

Merke

Die Folgen des Schlaganfalls - hohe Mortalität und bleibende Behinderungen - erfordern Konzepte, die allen Patienten raschen Zugang zu einer ausreichenden Versorgungsqualität verschaffen. 


\section{Evidenz in der Akutbehandlung}

Bei dem Großteil der Schlaganfälle (80\%) handelt es sich um einen sog. ischämischen Schlaganfall. Dabei verschließt ein Blutgerinnsel (Thrombus) ein dahinter liegendes Hirngefäß und es entwickelt sich ein Sauerstoff- und Nährstoffmangel in den betroffenen Arealen des Gehirns. In $20 \%$ der Fälle liegt eine Hirnblutung, ein sog. hämorrhagischer Schlaganfall, vor. Hierbei kommt es zu einer Zerreißung von Schlagadern des Gehirns. Das ins Gewebe austretende Blut führt durch eine Druckschädigung ebenfalls zu einer dauerhaften Schädigung des Nervengewebes.

Die optimale akute Therapie beruht derzeit auf 5 evidenzbasierten Behandlungsmethoden (s. „Therapie - Evidenzbasierte Schlaganfallbehandlung“).

\section{THERAPIE}

\section{Evidenzbasierte Schlaganfallbehandlung}

- Behandlung auf einer spezialisierten Schlaganfallstation, einer sog. „Stroke Unit“ [2]; Ziele sind:

- optimale Überwachung der Vitalparameter

- frühzeitige Schluckdiagnostik

- frühzeitiger Beginn von Ergo- und Physiotherapie

- frühzeitige Erkennung von Komplikationen oder Schlaganfallprogression

- rasche Einleitung von Diagnostik und medikamentösen Therapien

- frühzeitige Therapie mit Azetylsalizylsäure (ASS) zur Thrombozytenaggregationshemmung und somit Verhinderung weiterer Schlaganfälle [3,4]

- systemische Thrombolysetherapie mit „rekombinantem Tissue Plasminogen Activator“ (rt-pa) in den ersten 4,5 Stunden nach einem Schlaganfall mit dem Ziel, das gefäßverschließende Blutgerinnsel aufzulösen [5,6]

- Hemikraniektomie (teilweise und vorübergehende Schädelknochenentfernung zur Druckentlastung des gesunden Gehirngewebes) bei großen raumfordernden Infarkten $[7,8]$

- mechanische Rekanalisation (Thrombektomie), d. h. Entfernung großer Blutgerinnsel in Hirnarterien mittels eines Katheters [9-13]

Besonders die kausalen Therapien (Thrombolyse und Thrombektomie) sind hochgradig zeitkritisch. Es gilt das Motto „Time is Brain“: In jeder unbehandelten Minute nach Einsetzen der Durchblutungsstörung sterben durchschnittlich 1,9 Mio. Nervenzellen ab [14]. Jede Minute, die später behandelt wird, kostet einen weiteren Tag ohne Behinderung [15]. Daher müssen nicht nur die
Strukturen zur Umsetzung der evidenzbasierten Therapien geschaffen werden, die Patienten müssen diese Strukturen auch schnell erreichen können.

Die Telemedizin ist eine Möglichkeit, die Behandlung wohnortnah aufzubauen und den Mangel an vaskulären Neurologen in ländlichen Gebieten auszugleichen. Von Levine und Gorman wurde in einer Zukunftsvision bereits 1999 beschrieben, wie solch eine dezentrale telemedizinische Schlaganfallversorgung aussehen könnte [16]. Nach deren Konzept sollte der Schlaganfallpatient per Videokamera und mit Unterstützung eines ärztlichen Kollegen vor Ort durch einen vaskulär spezialisierten Neurologen untersucht werden. Ferner sollten die in der aufnehmenden Klinik angefertigten ComputertomografieAufnahmen des Gehirns dem entfernten Neurologen digital zur Verfügung gestellt werden. In Zusammenschau der telemedizinischen Untersuchung und der Bildbeurteilung sollte der Spezialist den behandelnden Ärzten vor Ort die Empfehlungen zur weiteren Diagnostik und Therapie geben können.

Merke

Thrombolyse und Thrombektomie sind hochgradig zeitkritische Verfahren, die für betroffene Patienten schnellstmöglich verfügbar sein sollten.

\section{Versorgungsprobleme}

Ende der 1990er-Jahre wurden die ersten Stroke Units gegründet und die Lysetherapie eingeführt. Die Diskrepanz in der Versorgungsqualität von Schlaganfallpatienten im ländlichen im Vergleich zum städtischen Bereich wurde dadurch zunehmend offensichtlicher.

Dies galt insbesondere auch für die ländlich geprägte Region Süd-Ost-Bayern: 2002 standen in einem Gebiet von ca. 25000 km² mit etwa 5 Mio. Einwohnern nur in den Ballungszentren München, Regensburg und Passau Stroke Units zur Verfügung. Nur ein geringer Teil der Schlaganfallpatienten aus dieser Region konnte folglich auf einer der spezialisierten Stationen behandelt werden (unter $20 \%)$. Der Großteil der Patienten wurde hingegen weiterhin in den internistischen Abteilungen der regionalen Krankenhäuser versorgt. Dort erfolgte keine Therapie auf einer speziellen Station und es bestand auch nicht die Möglichkeit der systemischen Thrombolysetherapie.

\section{Projekt „TEMPiS“}

\section{Gründung von „TEMPiS“}

Um diesem Versorgungsgefälle entgegenzuwirken, wurde Anfang 2002 ein neuartiges Konzept entwickelt. Den Versorgungskliniken der Region Süd-Ost-Bayern sollte die Schlaganfallexpertise aus den Städten im Rahmen 
eines telemedizinischen Netzwerks verfügbar gemacht werden durch

- eine rund um die Uhr verfügbare telemedizinische Konsultationsmöglichkeit,

- kontinuierliche zentrale und dezentrale Schulungen und Visitationen,

- den Aufbau von Stroke Units in allen regionalen Kooperationskliniken und

- die Erstellung und regelmäßige Aktualisierung definierter Behandlungsstandards.

Das „Telemedizinische Projekt zur integrierten Schlaganfallversorgung in der Region Süd-Ost-Bayern“ - kurz TEMPiS - startete im Februar 2003 als Pilotprojekt. Initial schlossen sich 12 regionale Kliniken dem Projekt an: die Krankenhäuser in Bad Tölz, Burglengenfeld, Cham, Dachau, Ebersberg, Eggenfelden, Freising, Kelheim, Mühldorf, Pasing, Rosenheim und Straubing.

In all diesen Krankenhäusern wurden umgehend Schlaganfalleinheiten aufgebaut. Diese sind räumlich abgetrennt, organisatorisch an die jeweilige internistische Abteilung angegliedert und werden von einem definierten ärztlich-pflegerisch-therapeutischen Team betreut. Bereits im 1. Jahr wurden fast 2000 Telekonsile durchgeführt ( $\triangleright$ Abb. 7). Diese Zahl wurde in den folgenden Jahren kontinuierlich gesteigert, was TEMPiS zu einem der größten telemedizinischen Schlaganfall-Netzwerke weltweit werden ließ.

Die Verbesserung der Versorgung konnte eindrucksvoll durch eine wissenschaftliche Effizienzanalyse belegt werden. Diese im Jahr 2006 veröffentlichte Studie schloss mehr als 3000 Patienten ein und zeigte: Patienten, die in TEMPiS-Kliniken behandelt wurden, hatten eine deutlich und signifikant bessere Prognose als Patienten, deren Therapie in vergleichbar ausgestatteten Kliniken ohne Projektanbindung stattfand [17] ( $\triangleright$ Abb. 3).

Merke

Die neuen Versorgungsstrukturen im TEMPiS-Netzwerk senkten für die behandelten Schlaganfallpatienten einer TEMPiS-Klinik signifikant das Risiko, die Klinik mit einer bleibenden Behinderung zu verlassen.

\section{Fünf Säulen des Netzwerks}

Aus dem oben genannten Konzept des Netzwerks heraus entwickelten sich die bis heute gültigen 5 Säulen des Netzwerks.

\section{Aufbau von Stroke Units}

Jede der inzwischen 19 Netzwerkkliniken hat sich bei Aufnahme ins Netzwerk verpflichtet, eine Stroke Unit mit dem klinischen Schwerpunkt der Schlaganfallversorgung aufzubauen. Ein weiterer fachlicher Schwerpunkt ist für diese Stationen nicht vorgesehen. Auf diesen Stroke Units, die neurologisch oder internistisch geführt wer-

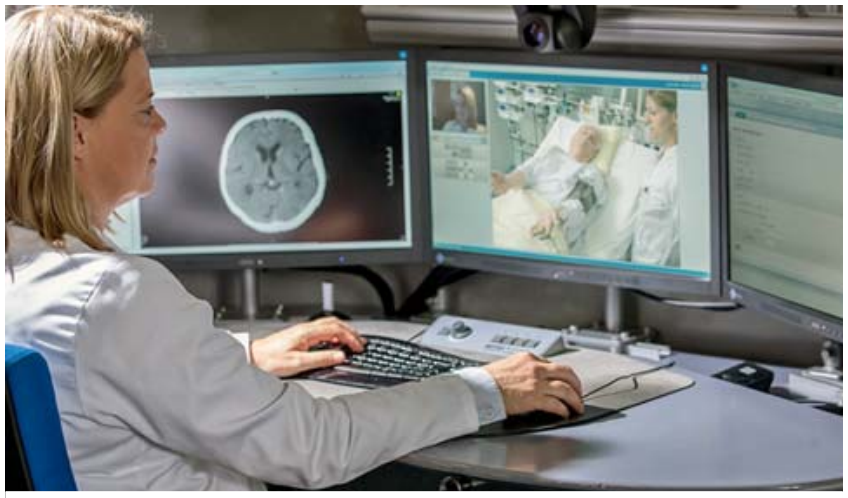

- Abb. 2 Telekonsilärztin im Zentrum München während einer Videokonferenz. Quelle: TEMPiS.

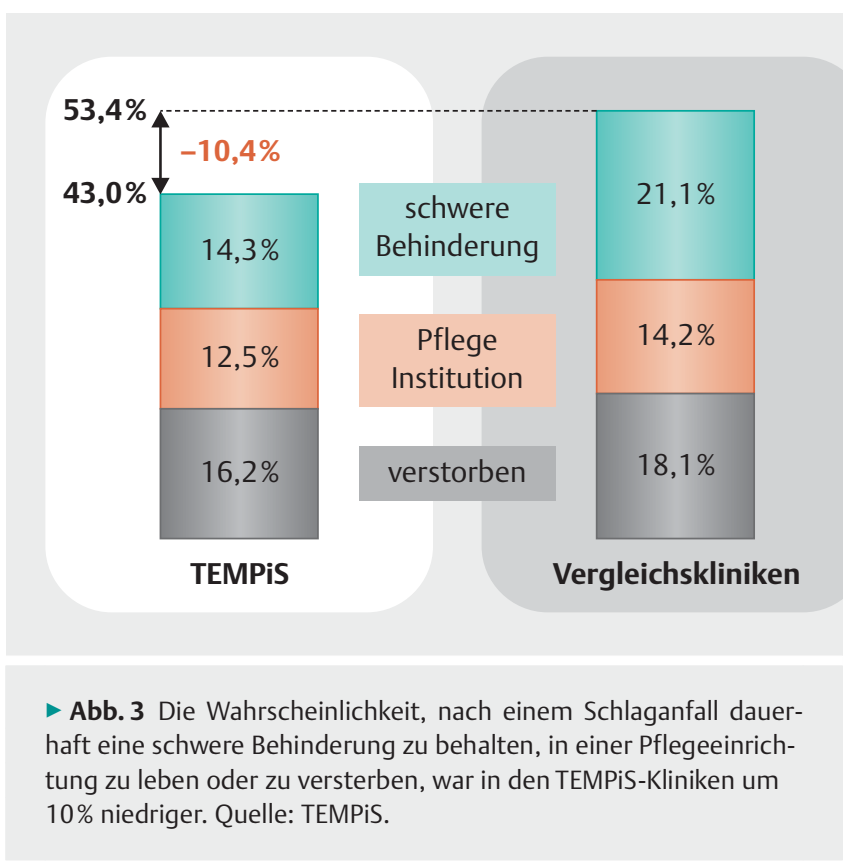

den, findet die übliche Diagnostik und Therapie des Schlaganfalls gemäß den aktuellen Standards statt. Hierzu gehören:

- Monitoring der Vitalparameter,

- Verfügbarkeit von CT und CT-Angiografie rund um die Uhr,

- regelmäßige ärztliche Untersuchungen,

- spezialisiertes therapeutisches Personal (Ergotherapie, Logopädie, Physiotherapie).

Neben den regelmäßigen ärztlichen Untersuchungen durch die Stationsärzte findet auch eine tägliche Visite der Monitorpatienten durch einen Facharzt für Neurologie vor Ort statt. Der Sozialdienst unterstützt das Team frühzeitig beim Entlassungsmanagement. 


\section{Schulungen - zentral und vor Ort}

Das Fortbildungsangebot ist ein zentraler Bestandteil des TEMPiS-Netzwerks. Hier sind alle Therapeutengruppen involviert. Von ärztlicher Seite findet 2-mal jährlich eine Visite jeder Kooperationsklinik statt. Dabei können neben medizinischen Fragestellungen ggf. auch strukturelle Fragen und organisatorische Abläufe unter Berücksichtigung der speziellen lokalen Gegebenheiten angesprochen werden. Fest im TEMPiS-Team angestellte Therapeuten (Logopädie, Ergotherapie, Physiotherapie) visitieren jede Klinik 2-mal jährlich und halten Fortbildungen zu spezifischen Themen vor Ort ab. Auch TEMPiS-Pflegekräfte der Zentren fahren mindestens je 5-mal jährlich in die Kliniken und schulen die Kollegen vor Ort.

Zusätzlich finden noch zentrale berufsgruppenspezifische und übergreifende Treffen, Fortbildungen und Konvents statt.

\section{Zusatzinformation}

\section{Regelmäßig stattfindende TEMPiS-Kurse}

- für Assistenzärzte: Schlaganfall-Intensivkurs ganztägig 2-mal/Jahr

- für die Pflege: Tele-Stroke-Nurse-Kurs 3-tägig 3-mal/Jahr

- für die Therapeuten: Therapeutentag ganztägig 1-mal/Jahr (meist im November)

- für alle Teilnehmer des Netzwerks, vorwiegend ärztliches Personal: sog. TEMPiS-Update 2-mal 1 Nachmittag/Jahr

\section{Standardisierte Behandlungsprotokolle}

Von Beginn an wurde ein gemeinsames Vorgehen für bestimmte Fragestellungen und Abläufe definiert. Diese heute als „Standardisierte optimierte Prozeduren“, TEMPiS-SOP, bekannten Standards orientieren sich am neuesten Stand der Wissenschaft und den Leitlinien der Fachgesellschaften. Sie sind öffentlich zugänglich (www.tempis. de) und werden regelmäßig, spätestens alle 2 Jahre, aktualisiert.

\section{Qualitätssicherung}

Neben der Entwicklung der SOP war die Qualitätssicherung von Anfang an ein wesentliches Kernmerkmal im Netzwerk. So verpflichten sich alle Kooperationskliniken zur Teilnahme am Schlaganfallregister der Bayerischen Arbeitsgemeinschaft für Qualitätssicherung (BAQ). Die BAQ-Daten aller TEMPiS-Kliniken werden jährlich ausgewertet und dann den Kliniken nochmals individuell rückgemeldet. Außerdem werden im Netzwerk ein Konsil- und ein Lyseregister geführt und ausgewertet, aus denen sich z. B. zeitliche Abläufe bei Lysepatienten und Lyseraten berechnen lassen. Aus den wissenschaftlichen Auswertungen der innerhalb des TEMPiS-Netzwerks gewon- nenen Daten gingen bisher mehr als 30 Publikationen in Fachzeitschriften hervor.

\section{Telemedizinische Versorgung}

Der Telekonsildienst ist an jedem Tag im Jahr rund um die Uhr über eine feste Telefonnummer erreichbar. Er wird im wöchentlichen Wechsel durch die Universitätsneurologie Regensburg und die Klinik für Neurologie im Klinikum Harlaching abgeleistet. Während seines Telekonsildiensts ist der Konsilarzt von weiteren klinischen Tätigkeiten freigestellt. Alle im Telekonsildienst eingesetzten Kollegen sind erfahrene Ärzte mit einem Schwerpunkt in vaskulärer Neurologie. Sowohl im Klinikum Harlaching als auch in der Universitätsneurologie in Regensburg wird der Telekonsildienst von einem Team aus jeweils etwa 8 Ärzten geleistet. Aufgrund der stetig wachsenden Anzahl an Telekonsilen wurde im Jahr 2012 ein zusätzlicher Telekonsildienst in den stark frequentierten Nachmittagsund Abendstunden eingeführt.

Der Telekonsilarzt hat an seinem Arbeitsplatz die Möglichkeit, über eine Audio-Video-Konferenz in ein speziell eingerichtetes Konsilzimmer in den Kooperationskliniken geschaltet zu werden ( $\bullet$ Abb. 2). So kann er dort den Patienten gemeinsam mit dem diensthabenden Kollegen vor Ort noch einmal anamnestizieren und untersuchen. Außerdem werden dem Konsilarzt die radiologischen Bilder wie kraniale CT, kraniale MRT oder CT-Angiografie der hirnversorgenden Gefäße zur Verfügung gestellt. So kann anhand der vorliegenden Befunde das weitere Vorgehen gemeinsam mit den Kollegen vor Ort entschieden werden ( $\triangleright$ Abb. 4). Bei Bedarf können weitere Untersuchungen in den Kooperationskliniken erfolgen oder auch eine Weiterverlegung des Patienten in ein größeres Zentrum veranlasst werden.

\section{Merke}

Neben der telemedizinischen Versorgung rund um die Uhr sieht das TEMPiS-Konzept regelmäßige Visiten, Fortbildungen, Qualitätssicherung und die Einhaltung von standardisierten Behandlungsprotokollen vor.

\section{Weitere Entwicklung des Netzwerks}

Die oben genannte Effizienzanalyse von 2006 [17] zeigte: Die Wahrscheinlichkeit, eine bleibende Behinderung zu erleiden oder an einem Schlaganfall zu versterben, wurde in einer TEMPiS-Klinik deutlich reduziert. Daher wurde TEMPiS im Jahre 2006 in die Regelversorgung der bayerischen Krankenkassen übernommen und wird seither als ein im Bayerischen Krankenhausplan verankertes Netzwerk weitergeführt.

Eine weitere Analyse der o.g. Daten unter medizinökonomischen Kriterien wies 2012 nach: Die akutstationäre Behandlung der Patienten war im TEMPiS-Netzwerk zwar teurer. Diese Mehrkosten wurden jedoch durch die langfristig geringere finanzielle Belastung der Pflegeversiche- 


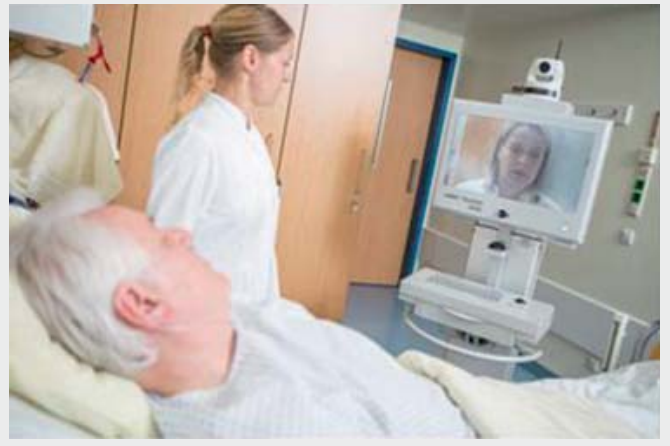

Patientenuntersuchung vor der Kamera

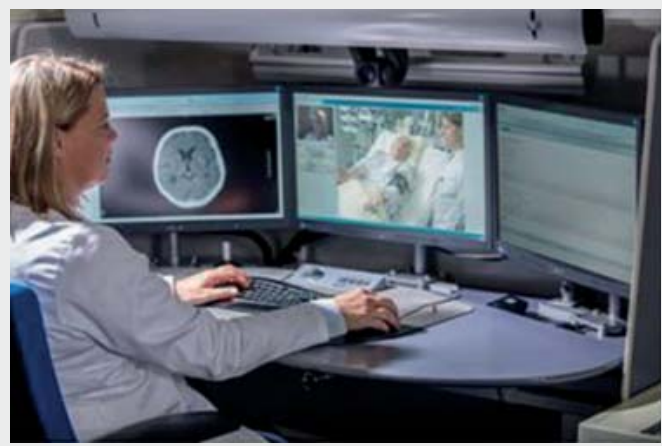

Besprechung des weiteren Vorgehens
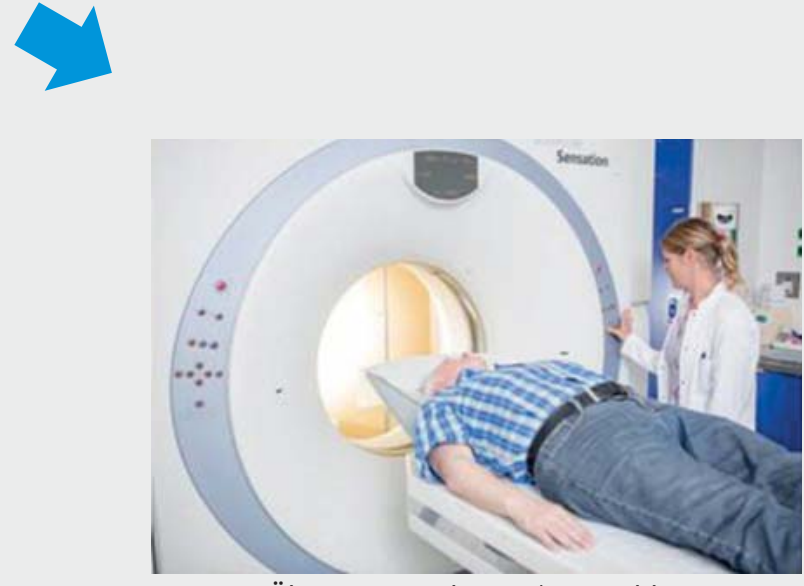

Übertragung der CT-/MRT-Bilder

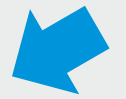

Abb. 4 Abläufe einer telekonsiliarischen Beurteilung. Quelle: TEMPiS.

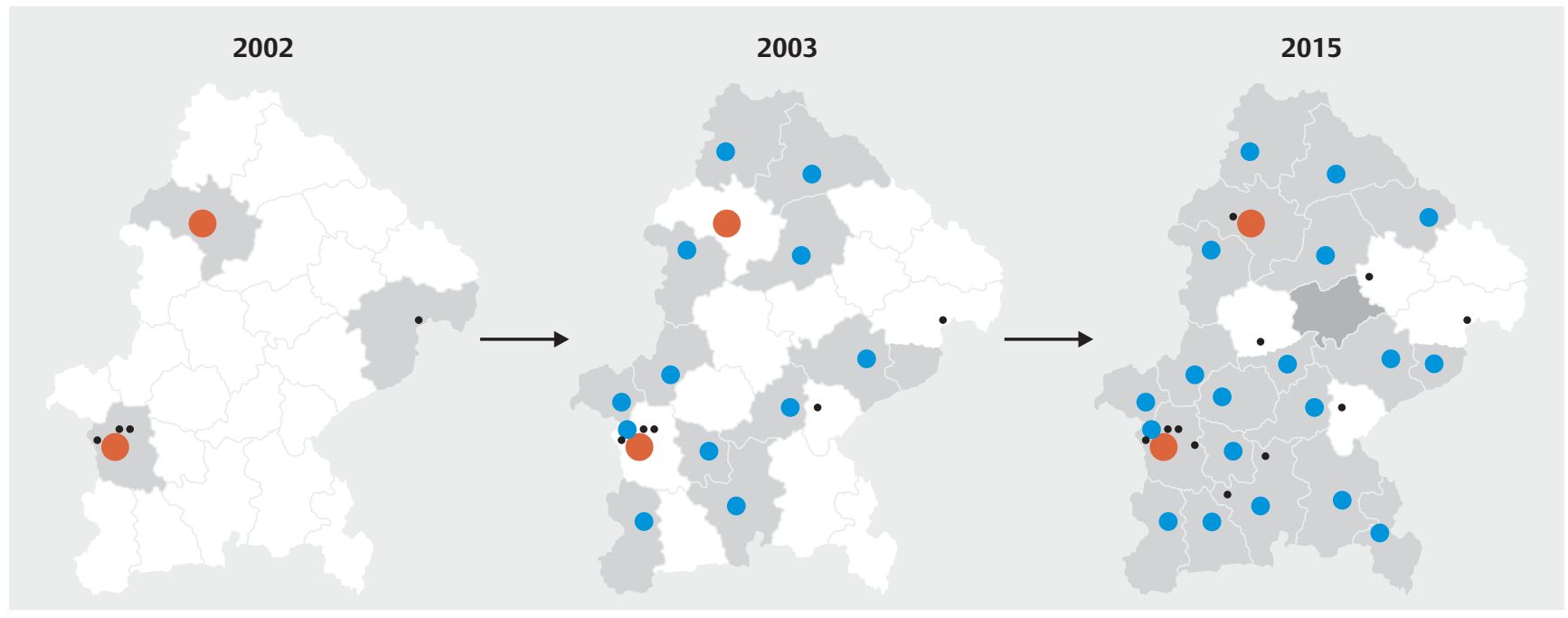

Abb. 5 Die Entwicklung des Netzwerkes. Quelle: TEMPiS. 


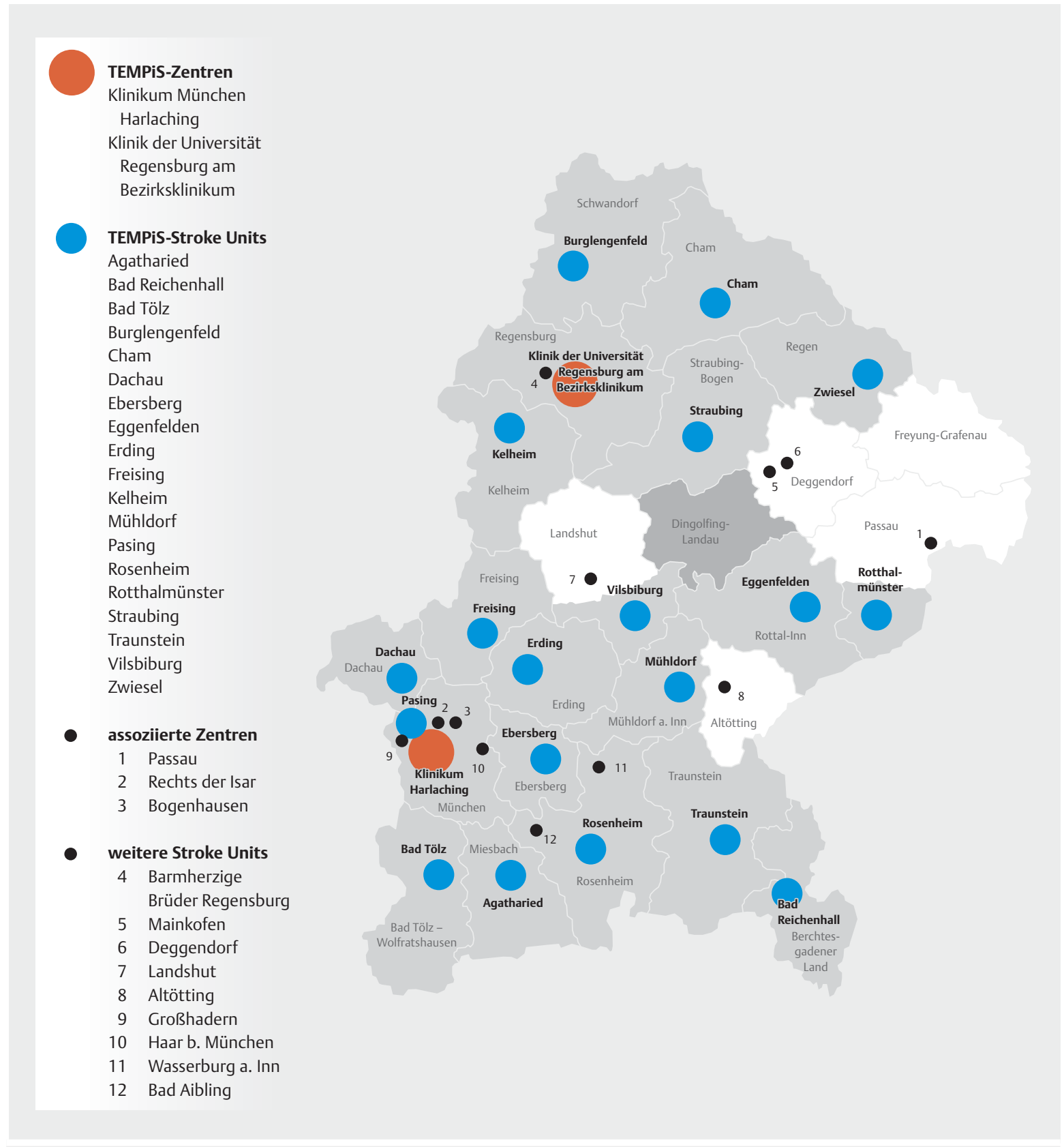

Abb. 6 Das TEMPiS-Netzwerk 2016. Rot: Zentren, blau: Kooperationskliniken, schwarz: weitere Neurologische Abteilungen. Quelle: TEMPiS.

rung aufgrund der weniger schwer ausgeprägten Behinderungen der im TEMPiS-Netzwerk behandelten Patienten kompensiert [18].

In den Jahren 2007-2015 wurden 7 weitere Kliniken Mitglieder des Netzwerks: Agatharied/Hausham, Bad Reichenhall, Erding, Rotthalmünster, Traunstein, Vilsbiburg und Zwiesel ( $\bullet$ Abb. 5). Die Stroke Units beider Zentrumskliniken sind als überregionale Stroke Units zertifiziert. Einige der Kooperationskliniken verfügen inzwischen über eigene neurologische Fachabteilungen mit zertifizierten regionalen Stroke Units. Drei der Tele-Stroke-Units sind ebenfalls nach den Kriterien der Deutschen SchlaganfallGesellschaft (DSG) zertifiziert ( $\triangleright$ Abb. 6). 


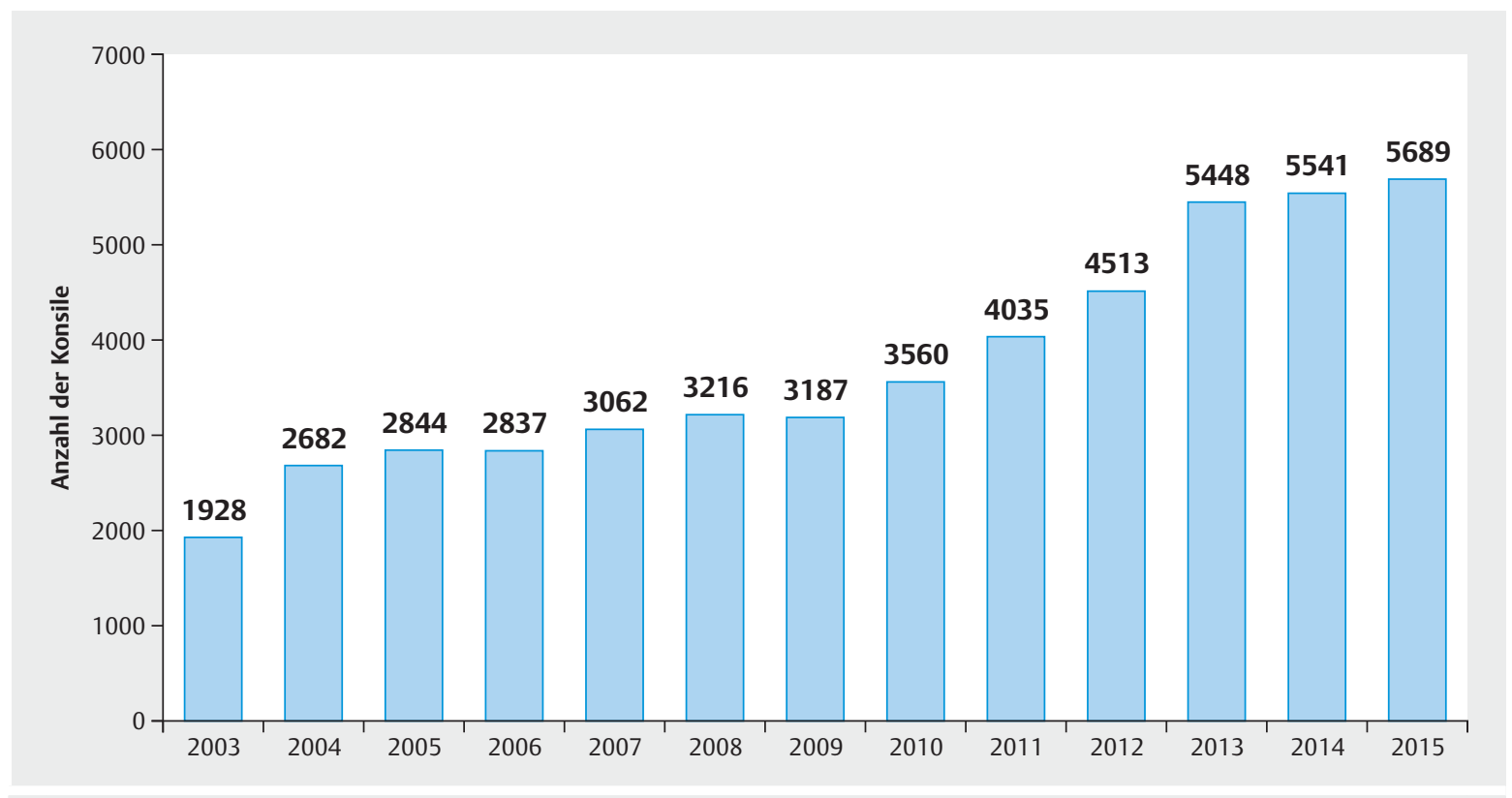

- Abb. 7 Anzahl der Telekonsile nach Jahren. Quelle: TEMPiS.

\section{Konsultationen und Lyserate}

Der kontinuierlich verfügbare Telekonsildienst hat seit Projektbeginn inzwischen über 50000 Telekonsultationen durchgeführt. Aktuell erfolgen ungefähr 5600 Konsultationen jedes Jahr, d.h. etwa 15 Telekonsile täglich ( $\bullet$ Abb. 7).

Der Anteil der Patienten mit akutem ischämischem Schlaganfall, welche die hochwirksame systemische Thrombolysetherapie erhalten, liegt in den TEMPiS-Kooperationskliniken mittlerweile bei ca. 17\% ( $>$ Abb. 8). Diese Thrombolysetherapie wird heute oft nach einer recht kurzen Zeit verabreicht. Im 1. Jahr nach Netzwerkbeginn vergingen noch 80 min vom Eintreffen im Krankenhaus (Door) bis zum Beginn der systemischen Lysetherapie (Needle). Durch viele Anstrengungen, Schulungen und strukturelle Veränderungen konnte diese DoorNeedle-Time (DNT) auf zuletzt wiederholt $35 \mathrm{~min}$ im Median (2013 und 2014) gesenkt werden [19].

\section{Merke}

Die Door-Needle-Zeit konnte im Netzwerk im Median auf 35 min reduziert werden.

In den Qualitätsindikatoren des bayernweiten Schlaganfallregisters (BAQ) erreichen die TEMPiS-Tele-StrokeUnits vergleichbar hohe Werte wie die anderen Kliniken in Bayern sowie alle vordefinierten Referenzwerte [20].

\section{ZUSATZINFORMATION}

\section{TEMPiS-Zentren}

- Städtisches Klinikum München, Klinikum Harlaching, Klinik für Neurologie und Neurologische Intensivmedizin

- medbo, Klinik und Poliklinik für Neurologie der Universität Regensburg am Bezirksklinikum Regensburg

\section{TEMPiS-Kliniken}

- Krankenhaus Agatharied GmbH - Krankenhaus Agatharied

- Kliniken Südostbayern - Kreisklinik Bad Reichenhall

- Asklepios Stadtklinik Bad Tölz

- Asklepios Klinik Burglengenfeld

- Sana Kliniken des Landkreises Cham - Krankenhaus Cham

- Helios Amper-Klinikum Dachau

- Kreisklinik Ebersberg

- Klinikum Landkreis Erding - Klinikum Erding

- Rottal-Inn-Kliniken Eggenfelden

- Klinikum Freising

- Goldberg-Klinik Kelheim

- Kliniken Kreis Mühldorf am Inn - Klinik Mühldorf

- Helios Klinikum München West - Klinikum Pasing

- RoMed Kliniken - RoMed Klinikum Rosenheim

- Krankenhaus Rotthalmünster

- Klinikum St. Elisabeth Straubing

- Kliniken Südostbayern - Klinikum Traunstein

- LaKUMed - Krankenhaus Vilsbiburg

- Arberlandkliniken Kommunalunternehmen - Arberlandklinik Zwiesel 


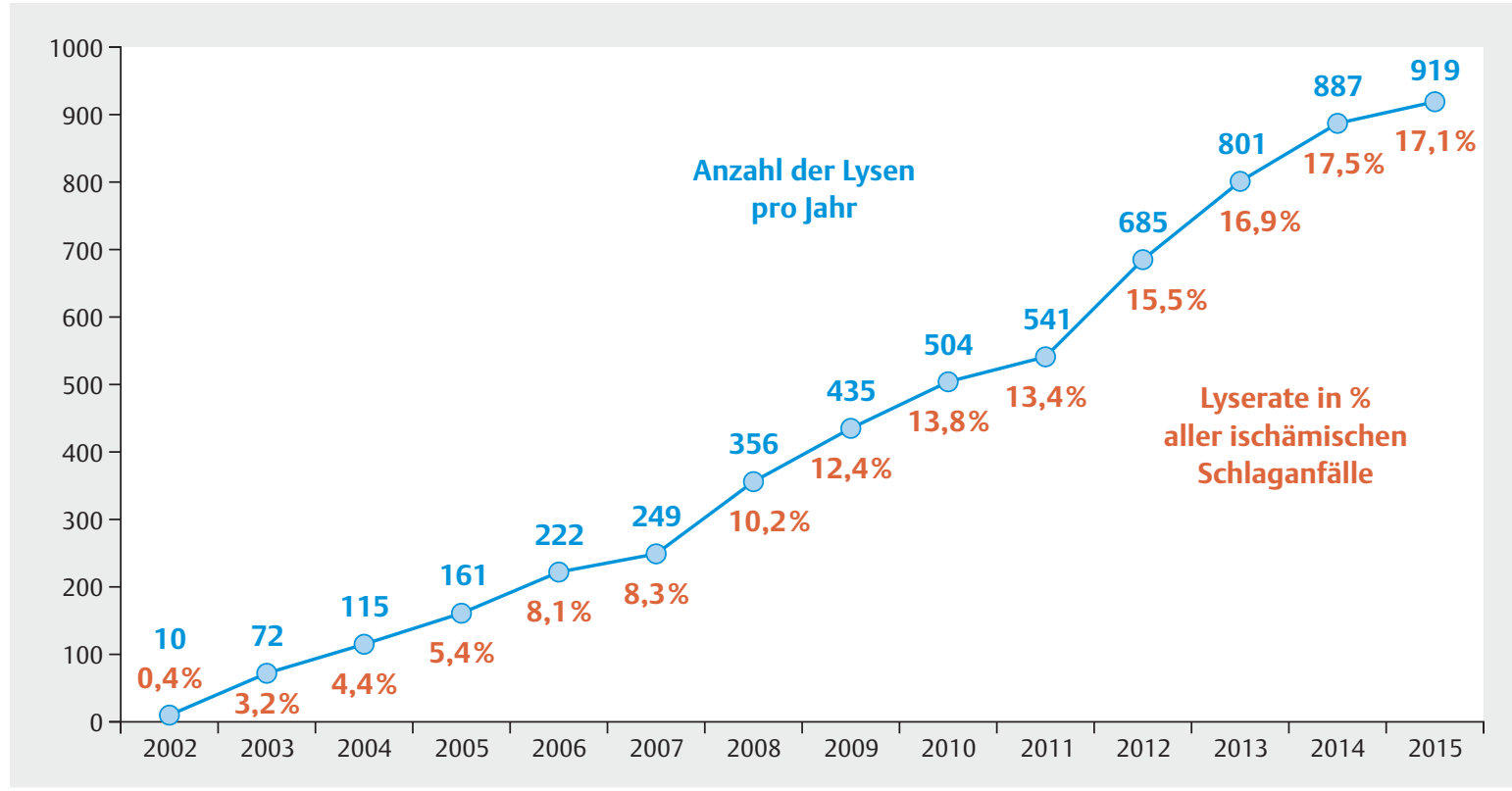

- Abb. 8 Anteil der Thrombolysen an allen ischämischen Schlaganfällen im Netzwerk. Quelle: TEMPiS.

Weitere Informationen wie z. B. die ausführlichen Jahresberichte und die Kontaktdaten der Projektbeteiligten finden Sie auch unter www.tempis.de.

\section{Finanzierung}

Seit 2006 ist TEMPiS Teil der Regelversorgung der Bayerischen Krankenkassen. Anfänglich über einen Sonderzuschlag finanziert, konnte 2012 die Finanzierung in das DRG-System integriert werden. Die Kooperationskliniken können ihre Kosten durch eine „telemedizinisch unterstützte“ Schlaganfallprozedur (OPS 8.98b, sog. „andere neurologische Komplexbehandlung des akuten Schlaganfalles“) decken. Die Kosten der Zentren (Telekonsildienste, ärztliche, pflegerische, therapeutische Koordination, Qualitätssicherung etc.) werden weiterhin über einen patientenbezogenen Sonderzuschlag finanziert.

\section{Andere Netzwerke}

In den letzten 10 Jahren wurden in zahlreichen Regionen ähnlich strukturierte Schlaganfall-Netzwerke wie TEMPiS aufgebaut.

\section{ZUSATZINFORMATION}

Weitere Schlaganfall-Netzwerke

- Nord-Ost-Bayern (STENO)

- Süd-West-Bayern (NEVAS)

- Sachsen (SOS-NET)

- Augsburger Raum (TESAURUS)

- Hessen (Neuro-Netz Mitte)

- Thüringen (SATELIT)

- Rheinland-Pfalz (Beginn April 2016: Telestroke-Netzwerk Rheinland-Pfalz)

- Niedersachsen (Beginn Mai 2016: Telestroke-Netzwerk der MH Hannover)

\section{Schlussfolgerung}

Das telemedizinische Schlaganfall-Netzwerk TEMPiS hat es über viele Jahre geschafft, die Schlaganfallversorgung in den ländlichen Regionen Süd-Ost-Bayerns erheblich zu verbessern und auf ein hohes Qualitätsniveau anzuheben. Die Versorgungslücke für die 2,4 Mio. Einwohner konnte somit geschlossen werden. TEMPiS gehört zu den größten und führenden Tele-Schlaganfall-Netzwerken in Europa. 


\section{KERNAUSSAGEN}

- Jeder akute Schlaganfall sollte - wenn möglich auf einer Stroke Unit von einem spezialisierten

Team versorgt werden.

- Systemische Thrombolyse und mechanische Thrombektomie sind - wie die Therapie auf einer Stroke Unit - evidenzbasierte Verfahren, die dem Patienten schnellstmöglich zur Verfügung gestellt werden sollten.

- Um die Versorgungslücke von Patienten zu schließen, die bisher nicht in Stroke Units behandelt werden konnten, wurden in den letzten Jahren zahlreiche Schlaganfall-Netzwerke gegründet. Sie versorgen Patienten im ländlichen Raum durch sog. Tele-Stroke-Units.

- TEMPiS war eines der ersten telemedizinischen Schlaganfall-Netzwerke weltweit und stellt derzeit mit ca. 5600 Konsilen jährlich eines der größten Netzwerke der Welt dar.

- In 19 Netzwerkkliniken werden durch die 2 Zentren in München-Harlaching und Regensburg mehr als 2 Mio. Einwohner der Region Süd-Ost-Bayern versorgt.

- Neben Telekonsildienst und regelmäßigen Schulungen im ärztlichen Bereich liegt ein Fokus im Netzwerk auf der interdisziplinären Zusammenarbeit von Ärzten, Pflege, Physiotherapie, Ergotherapie und Logopädie.

- Die 5 Säulen des Netzwerks sind: Aufbau von Stroke Units, regelmäßige Fortbildungen, standardisierte Prozeduren (SOP), Qualitätssicherung und ein rund um die Uhr verfügbarer Telekonsildienst.

- Die Behandlung auf einer Tele-Stroke-Unit reduziert signifikant das Risiko, eine bleibende Behinderung durch den Schlaganfall zu erleiden.

- Die im Netzwerk entstehenden höheren stationären Behandlungskosten werden durch langfristig geringere Kosten der Pflegeversicherung kompensiert.

\section{Interessenkonflikt}

Die Autoren erklären, dass keine Interessenkonflikte vorliegen.

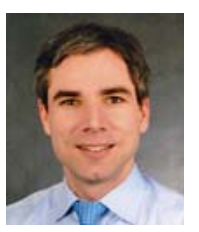

\section{Dr. med. Nicolas Völkel}

ist Facharzt an der Klinik für Neurologie und Neurologische Intensivmedizin des Klinikums Harlaching. Seit 2014 koordiniert er das TEMPiS-Netzwerk für den Standort München-Harlaching.

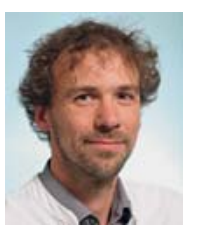

\section{Dr. med. Gordian J. Hubert}

ist Oberarzt der Klinik für Neurologie und Neurologische Intensivmedizin des Klinikums Harlaching. Er ist zudem Gesamtkoordinator des TEMPiS-Netzwerks.

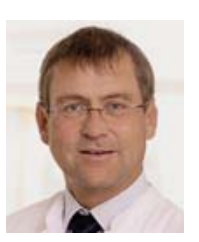

\section{Prof. Dr. med. Roman L. Haberl}

ist Chefarzt der Klinik für Neurologie und Neurologische Intensivmedizin des Klinikums Harlaching.

\section{Korrespondenzadresse}

\section{Dr. med. Nicolas Völkel}

Klinik für Neurologie und Neurologische Intensivmedizin Klinikum Harlaching

Sanatoriumsplatz 2

81545 München

nicolas.voelkel@klinikum-muenchen.de

\section{Literatur}

[1] Heuschmann PU, Busse O, Wagner M et al. Schlaganfallhäufigkeit und Versorgung von Schlaganfallpatienten in Deutschland. Akt Neurol 2010; 37: 333-340

[2] Stroke Unit Trialists' Collaboration. Organised inpatient (stroke unit) care for stroke (Review). Cochrane Database Syst Rev 2013; (9): CD000197

[3] International Stroke Trial Collaborative Group. The International Stroke Trial (IST): a randomised trial of aspirin, subcutaneous heparin, both, or neither among 19435 patients with acute ischaemic stroke. Lancet 1997; 349: 1569-1581

[4] CAST (Chinese Acute Stroke Trial) Collaborative Group. CAST: randomised placebo-controlled trial of early aspirin use in 20,000 patients with acute ischaemic stroke. Lancet 1997; 349: 1641-1649

[5] Lees KR, Bluhmki E, von Kummer $R$ et al. Time to treatment with intravenous alteplase and outcome in stroke: an updated pooled analysis of ECASS, ATLANTIS, NINDS and EPITHET trials. Lancet 2010; 375: 1695-1703

[6] Emberson J, Lees KR, Lyden P et al. Effect of treatment delay, age, and stroke severity on the effects of intravenous thrombolysis with alteplase for acute ischaemic stroke: a meta-analysis of individual patient data from randomised trials. Lancet 2014; 384: 1929-1935

[7] Vahedi K, Hofmeijer J, Juettler E et al. Early decompressive surgery in malignant infarction of the middle cerebral artery: a pooled analysis of three randomised controlled trials. Lancet Neurol 2007; 6: 215-222 
[8] Juettler E, Unterberg A, Woitzik J et al. Hemicraniectomy in older patients with extensive middle-cerebral-artery stroke (DESTINY II: DEcompressive Surgery for the Treatment of malignant INfarction of the middle cerebral arterY II). N Engl J Med 2014; 370: 1091-1100

[9] Berkhemer OA, Fransen PS, Beumer D et al. A randomized trial of intraarterial treatment for acute ischemic stroke. $\mathrm{N}$ Engl J Med 2015; 372: 11-20

[10] Campbell BC, Mitchell PJ, Kleinig T] et al.; EXTEND-IA Investigators. Endovascular therapy for ischemic stroke with perfusion-imaging selection. N Engl J Med 2015; 372: 1009-1018

[11] Jovin TG, Chamorro A, Cobo E et al.; REVASCAT Trial Investigators. Thrombectomy within 8 hours after symptom onset in ischemic stroke. N Engl J Med 2015; 372: 2296-2306

[12] Goyal M, Demchuk AM, Menon BK et al.; ESCAPE Trial Investigators. Randomized assessment of rapid endovascular treatment of ischemic stroke. N Engl J Med 2015; 372: 1019-1030

[13] Saver ], Goyal M, Bonafe A et al. Stent-retriever thrombectomy after intravenous t-PA vs. t-PA alone in stroke. N Engl J Med 2015; 372: 2285-2295

[14] Saver J. Time is brain - quantified. Stroke 2006; 37: 263-266

[15] Meretoja A, Keshtkaran M, Saver JL et al. Stroke thrombolysis: save a minute, save a day. Stroke 2014; 4: 1053-1058
[16] Levine SR, Gorman M. "Telestroke": the application of telemedicine for stroke. Stroke 1999; 30: 464-469

[17] Audebert HJ, Schenkel J, Heuschmann PU et al. Effects of the implementation of a telemedical stroke network: the Telemedic Pilot Project for Integrative Stroke Care (TEMPiS) in Bavaria, Germany. Lancet Neurol 2006; 5: 742-748

[18] Schenkel J, Reitmeir P, von Reden S et al. Kostenanalyse telemedizinischer Schlaganfallbehandlung. Gesundheitswesen 2013; 75: 405-412

[19] Müller-Barna P, Hubert G], Boy S et al. TeleStroke units serving as a model of care in rural areas. 10-year experience of the TeleMedical project for integrative stroke care. Stroke 2014; 45: 2739-2744

[20] Müller-Barna P, Boy S, Hubert G] et al. Convincing quality of acute stroke care in Telestroke Units. Eur Res Telemed 2015; 75: $1-9$

Bibliografie

DOI http://dx.doi.org/10.1055/s-0042-108417

Anästhesiol Intensivmed Notfallmed Schmerzther 2017; 52: 127-136 @ Georg Thieme Verlag KG Stuttgart · New York ISSN 0939-2661 